\title{
Functional identification of the prnABCD operon and its regulation in Serratia plymuthica
}

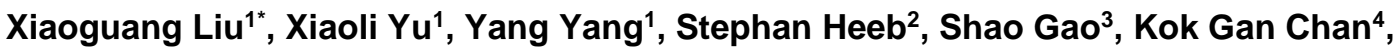 \\ Miguel Cámara², Kexiang Gao ${ }^{5}$
}

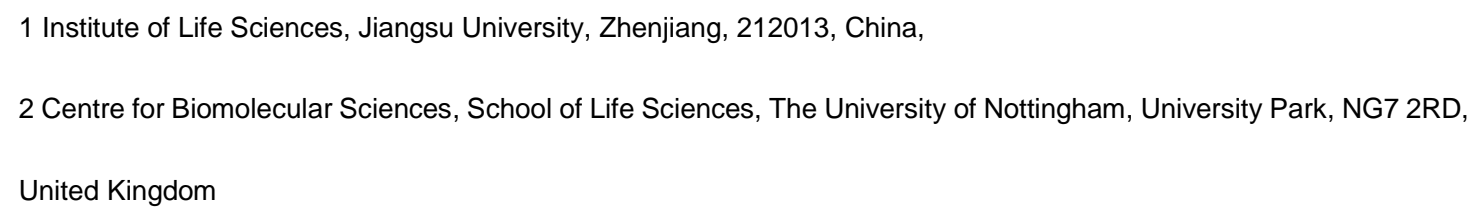

\section{Abstract}

The antibiotic pyrrolnitrin (PRN) is a tryptophan-derived secondary metabolite that plays an important role in the biocontrol of plant diseases due to its broad-spectrum of antimicrobial activities. The PRN biosynthetic gene cluster remains to be characterized in Serratia plymuthica, though it is highly conserved in PRN-producing bacteria. To better understand PRN biosynthesis and its regulation in Serratia, the prnABCD operon from S. plymuthica G3 was cloned, sequenced and expressed in Escherichia coli DH5a. Furthermore, an engineered strain prnind which is a conditional mutant of G3 prnABCD under the control of the Ptac promoter was constructed. This mutant was able to overproduce PRN with isopropylthiogalactoside (IPTG) induction by over-expressing $\operatorname{prn} A B C D$, whilst behaving as a conditional mutant of G3 prnABCD in the absence of IPTG. These results confirmed that prnABCD is responsible for PRN biosynthesis in strain G3. Further experiments involving 
Iux-/dsRed-based promoter fusions, combined with site-directed mutagenesis of the putative $\sigma^{S}$ extended -10 region in the prnA promoter, and liquid chromatography-mass spectrometry (LC-MS) analysis extended our previous knowledge about G3, revealing that quorum sensing (QS) regulates PRN biosynthesis through cross talk with RpoS, which may directly activated prnABCD transcription. These findings suggest that PRN in S. plymuthica $\mathrm{G} 3$ is produced in a tightly controlled manner, and has diverse functions, such as modulation of cell motility, in addition to antimicrobial activities. Meanwhile, the construction of inducible mutants could be a powerful tool to improve PRN production, beyond its potential use for the investigation of the biological function of PRN.

Keywords: Serratia plymuthica G3, the prnABCD operon, a conditional mutant, over-expressing, pyrrolnitrin biosynthesis and regulation 


\section{Introduction}

The antibiotic pyrrolnitrin [3-chloro-4-(2'-nitro-3'-chloro-phenyl) pyrrole, PRN] is a secondary metabolite derived from tryptophan that was originally isolated from Burkholderia pyrrocinia (Pseudomonas pyrrocinia) (Arima et al., 1964). PRN displays a broad-spectrum of antimicrobial activities against a number of plant and human pathogenic bacteria and fungi (Chernin et al., 1996; Di Santo et al., 1998; Kirner et al., 1998), in addition to its nematicidal activity which was reported recently (Nandi et al., 2015). Additionally, PRN biosynthesis in rhizobacteria is known to play a key role in bacterial survival (Costa et al., 2009), and in biological control and induced systemic resistance in plants (Kim et al., 2014). Therefore, natural derivative products of PRN can be used as synthetic lead compounds for the production of novel agricultural fungicides, such as fludioxonil and fenpiclonil as promising alternatives to synthetic pesticides in sustainable agricultural practices (Keum et al., 2009).

The prnABCD gene cluster, first described in Pseudomonas fluorescens BL915, encodes four enzymes PrnA, PrnB, PrnC and PrnD, which are responsible for PRN biosynthesis (Hammer et al., 1997). Previous studies have established that the bacterial ability to produce PRN greatly affects their biocontrol activity against plant pathogens; thus, a number of studies have been carried out to elucidate PRN production and its regulatory mechanisms in bacteria (Costa et al., 2009; Mozes-Koch et al., 2012). For example, comparative analysis of DNA and protein sequences of the prnABCD gene cluster in PRN-producing bacteria revealed that it is highly conserved among strains of the genera Pseudomonas, Burkholderia and Serratia (Hammer et al., 1999; Costa et al., 2009).

Apart from nutritional clues which are known to affect PRN biosynthesis in Burkhoderia spp. O33 and Pseudomonas chlororaphis O6 (Keum et al., 2009; Park et al., 2011), PRN production in bacteria has been well documented to be modulated by several global regulators. For instance, quorum sensing (QS) is a cell-to-cell communication system that allows bacteria to coordinate gene expression and therefore the behavior, of the entire community in a population-dependent manner using small diffusible signal molecules, such as $\mathrm{N}$-acyl homoserine lactones (AHLs) in gram-negative bacteria (Rutherford and Bassler, 2012). AHL signalling has been proven to control PRN biosynthesis in S. plymuthica (Liu et al., 2007) and 
the B. cepacia complex (Schmidt et al., 2009). Several other global regulators, including the master regulator of the general stress response RpoS $\left(\sigma^{S}\right)$ and the GacS/GacA two-component system (TCS) are also involved in controlling PRN biosynthesis in $S$. plymuthica IC1270 (Ovadis et al., 2004) and Pseudomonas protegens (P. fluorescens) Pf-5, although this strain is unable to produce AHLs (Sarniguet et al., 1995). However, whether this type of regulation applies generally, as well as the interactions among these regulators are still poorly understood. Furthermore, it is well known that the bacterial RpoD $\left(\sigma^{70}\right)$ subclass of promoters contains two conserved sequence elements where the -10 TATACT box is absolutely essential to start transcription in prokaryotes, whilst the -35 TTGACA box affects the transcription rate. Although the $\sigma^{S}$ and $\sigma^{70}$ subunits recognize very similar promoter sequences,

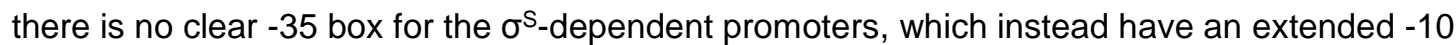
consensus sequence $\mathrm{KCTATACT}$ ( $\mathrm{K}=\mathrm{G} / \mathrm{T}$, conserved positions in bold) where a $\mathrm{T} / \mathrm{GC}$ motif at the $-14 /-13$ positions is very important (Becker and Hengge-Aronis 2001; Weber et al., 2005; Umarov and Solovyev, 2017). This raises the question of whether RpoS directly controls the promoter activity of $\operatorname{prn} A B C D$, as well as interactions with QS. This remains to be investigated to better understand the mechanisms behind the integrated networks modulating PRN biosynthesis.

S. plymuthica has been used as a biocontrol agent (BCA) against several phytopathogenic fungi due to its ability to produce multiple antifungal factors, including the antibiotic PRN (de Vleeschauwer and Höfte, 2007). We isolated the strain S. plymuthica G3 from wheat stem (Liu et al., 2010, and identified two Luxl homologues, Spll and Spsl, which are responsible for synthesis of various $\mathrm{AHL}$ signal molecules. Interestingly, both $\mathrm{AHL}$ signalling and stationary-phase RpoS are implicated in controlling biofilm formation and cell motility in G3 (Liu et al., 2011; Liu et al., 2016). Analysis of the 5'-flanking region of prnABCD using the CNNPromoter (Umarov and Solovyev, 2017) allowed us to identify a putative $\sigma^{70}-10$ box TAATCT overlapping with a potential $\sigma^{S}$ extended -10 element TCTAACAT (conserved positions in bold), similar to that found in E. coli (Weber et al., 2005), located at a position approximately 30 nucleotides upstream from the prnA start codon ATG. This finding implies that prnABCD might have an RpoS-dependent promoter, but this remains to be verified. In this 
study, the prnABCD operon from S. plymuthica was characterized for the first time, and the results show that it is responsible for PRN biosynthesis in strain G3. Assay of lux-based promoter fusions, combined with LC-MS suggested that PRN biosynthesis is tightly controlled through interactions between AHL signalling and RpoS. Further site-directed mutagenesis of the putative extended -10 region in the prnA promoter revealed that RpoS may directly activate prnABCD transcription. In addition, a conditional mutant prnind of $\mathrm{G} 3$ prnABCD was constructed using a gene replacement strategy under the control of the inducible Ptac promoter to improve PRN yield along with exploring the role of PRN in bacterial biology.

\section{Materials and methods}

Bacterial strains, plasmids, and growth conditions The bacterial strains, plasmids and primers used in this study are listed in Table 1. S. plymuthica G3 (CGMCC no. 4134) and its derivatives were grown at $30^{\circ} \mathrm{C}$, and Escherichia coli strains were grown at $37^{\circ} \mathrm{C}$ in Luria-Bertani broth (LB) or on LB agar plates. Antibiotics, when required, were added to the growth media at the following concentrations: ampicillin, $100 \mu \mathrm{g} / \mathrm{ml}$; gentamicin, $20 \mu \mathrm{g} / \mathrm{ml}$; kanamycin, $50 \mu \mathrm{g} / \mathrm{ml}$; and tetracycline, $25 \mu \mathrm{g} / \mathrm{ml}$. The pathogenic fungus Cryphonectria parasitica (ATCC 38755), the causal agent of chestnut blight, was routinely grown on potato dextrose agar (PDA) at $25^{\circ} \mathrm{C}$.

DNA preparation and manipulations Standard methods were used for plasmid and genomic DNA isolation, restriction enzyme digestion, agarose gel electrophoresis, ligation, and transformation (Ausubel et al., 1994), or the manufacturer's instructions were followed.

Cloning and heterologous expression of the G3 prnABCD operon S. plymuthica G3 is able to produce the antibiotic PRN (Liu et al., 2010). Searches within the G3 genome (Liu and Chan, unpublished data) identified a prnABCD operon. To clone the prnABCD gene cluster, PCR was performed using the primer pair prnA-F and prnD-R (Table 2), the G3 genomic DNA as template and LA-Taq (TaKaRa, Dalian, China) for amplification of the entire prnABCD 
operon with its promoter region including 574 nucleotides upstream of the start codon. The following program was used for thermal cycling: $94^{\circ} \mathrm{C}$ for $6 \mathrm{~min}$; followed by 30 cycles at $94^{\circ} \mathrm{C}$ for $1 \mathrm{~min}, 55^{\circ} \mathrm{C}$ for $1 \mathrm{~min}$, and extension at $72^{\circ} \mathrm{C}$ for $5 \mathrm{~min}$; and a final extension at $72^{\circ} \mathrm{C}$ for 10 min. After purification, the resulting ca.6-kb PCR product was cloned into the vector pMD19-T (TaKaRa) to generate the plasmid pPRN, and transformed into $E$. coli $\mathrm{DH} 5 \alpha$ for heterologous expression of the G3 prnABCD operon. Positive clones carrying the recombinant plasmid pPRN were selected by PCR and sequencing (Sangon Co. Ltd., Shanghai, China). The GenBank accession no. for the G3 prnABCD operon is JF274257.

Further phylogenetic analyses of 20 complete prnABCD sequences from Pseudomonas, Serratia and Burkholderia spp. currently available in the GenBank database, along with G3 prnABCD sequenced in this study, were carried out with prnBCDA from Myxococcus fulvus as the outgroup. A nucleic acid-based neighbour-joining tree was constructed, and a bootstrap test (1000 repetitions) was performed as previously described (Liu et al., 2011).

Construction of a conditional mutant prnind of G3 prnABCD To verify the biological role of the prnABCD gene loci, and obtain an engineered strain of $S$. plymuthica G3 with the ability to over-produce PRN, a G3 prnind conditional mutant was first constructed by replacing the native promoter of the prnABCD operon in the G3 chromosome with the lac/o repressor gene, along with the Ptac inducible promoter as described by Rampioni et al., with minor modifications (Rampioni et al, 2010). The primers used are listed in Table 2. Briefly, the construction of the conditional mutant was performed as follows (Fig. 1-a ): (a) a 662 bp fragment prn-LA, using as the left homologous arm from the upstream region of $p r n A B C D$ was amplified by PCR using the primer pair prnind1-F/R, and included the Sacl/BamHI sites for double digestion, (b) the 1264 bp fragment prn-RA, using as the right homologous arm carrying part of the prnA ORF was amplified by PCR using the primer pair prnind2-F/R and included the EcoRI site to allow cloning into pMD19-T for digestion with EcoRI, (c) the $853 \mathrm{bp}$ Gm cassette was excised from plasmid p34S-Gm with BamHI (Dennis and Zylstra, 1998) and inserted into the BamHI site between the two fragments prn-LA and prn-RA, and (d) the $1.5 \mathrm{~kb}$ lac/Q Ptac inducible promoter fragment was excised from pME6032 with BamHI/ EcoRI and, 
then inserted into the same sites of pBluescript II SK(-) between the Gm cassette and the right arm prn-RA. These fragments mentioned above were assembled into pBluescript II SK(-) sequentially to generate $\mathrm{pSK}-\mathrm{YX}$. After being digested $\mathrm{pSK}-\mathrm{YX}$ with Sacl/EcoRI, followed by Klenow blunting, the ca. 4300 bp fragment was ligated into the Smal site of pDM4 to generate the suicide plasmid pDM4-YX, which was then conjugated into the parent strain G3. The inducible mutant prnind was obtained through crossover of the insert carried by this recombinant vector pDM4-YX into the G3 chromosome, followed by a second crossover triggered upon $5-15 \%$ sucrose selection and the isolation of $\mathrm{Gm}^{\mathrm{R}}$ clones. The correct chromosomal insertion was verified by PCR and sequencing. Growth rates of the wild-type G3 and its inducible mutant, prnind, in the absence or presence of $0.5 \mathrm{mmol}$ IPTG were monitored every three hours at $\mathrm{OD}_{600} \mathrm{~nm}$.

PRN identification PRN was extracted from five-day-old bacterial cultures growing on PDA $+1 \%$ glycerol and incubated at $30^{\circ} \mathrm{C}$, followed by thin-layer chromatography (TLC) detection as previously described (Zhou et al., 2012) or LC-MS2 analysis as follows: an Agilent Technologies 1200 Series LC system (Waldbronn, Germany) was used for high-performance liquid chromatography (HPLC) analysis. The column oven was maintained at $50^{\circ} \mathrm{C}$. The HPLC column used was a Phenomenex Gemini C18 column $(3.0 \mu \mathrm{m}, 100 \times 3.0 \mathrm{~mm})$ with an appropriate guard column. Mobile phase A was water, and mobile phase B was acetonitrile. The flow rate throughout the chromatographic separation was $450 \mu \mathrm{l} / \mathrm{min}$. The binary gradient began initially at 10\% B and increased linearly to $99 \%$ B in 4.5 min. This composition was maintained for a further $1.5 \mathrm{~min}$, rapidly decreased to $10 \% \mathrm{~B}$ over $0.1 \mathrm{~min}$, and stayed at this composition for $2.9 \mathrm{~min}$. The total run time per sample was $10 \mathrm{~min}$. In total, $10 \mu \mathrm{l}$ of samples were injected per analysis. The MS system used was a Bruker HCT Plus ion trap mass spectrometer equipped with an electrospray ionisation (ESI) interface. The electrospray settings were as follows: nebuliser gas, 50 psi; drying gas, $9 \mathrm{~L} / \mathrm{min}$; and, drying temperature, $365^{\circ} \mathrm{C}$. MS analysis was conducted under negative electrospray conditions (-ES) with the MS in MRM (multiple reaction monitoring) mode to screen the LC eluent specifically for PRN by comparing LC retention time of detected peaks with the available synthetic standard. The 
precursor-product ion mass transition used for MRM detection was m/z 254.8-189.7.

Antifungal activity and cell motility Bioassays of the antifungal activity were performed in dual cultures on plates by measuring the diameter of the inhibition zone of bacterial strains against the chestnut blight pathogen C. parasitica as previously described (Liu et al., 2010). The bacterial swimming motility was assayed as described previously (Liu et al., 2016).

Construction and assay of Iux-based promoter fusions To determine whether RpoS or the QS system also regulates G3 prnABCD expression, a prnA::lux transcriptional fusion was constructed and assayed as described previously (Zhou et al., 2012). Briefly, a ca. 550 bp PCR fragment including the promoter region of the prnABCD operon was first cloned into pMD19-T, digested with Xbal/Sall, and ligated into the Smal site of pBluelux (Atkinson et al., 2008) after Klenow blunting. Positive clones were selected according to bioluminescence and sequencing. Next, the plasmid pBluelux/prnA::/ux was digested with Pstl and ligated into the same site of the broad-host-range vector pUCP26 to generate pPprnA::lux, which was used to transform E.coli /S17-1 to enable conjugation with the wild-type or the $\Delta r p o S$ mutant. A similar strategy was used to construct the lux-based promoter fusions pPrpoS::lux ( ca. 580 bp EcoRI fragment), pPspll::Iux ( ca. 520 bp Xbal/Sall fragment) and pPspsl::lux ( ca. 590 bp Xbal/Sall fragment) using the pair of primers rpoS-luxF/R, spll-luxF/R and sps/-luxF/R, respectively (Table 2).

Site-direct mutagenesis To unravel whether RpoS may directly control the transcription of prnABCD in strain G3, site-directed mutagenesis of four nucleotide positions conserved in the extended -10 region in the prnABCD promoter was conducted. All primers used below are listed in Table 2. Briefly, PCR amplification of the shorter prnA promoter PprnS (61 bp upstream from ATG) from G3 genomic DNA, and the fluorescent reporter DsRed from plasmid pDsRed2 (Clontech) was performed with the primer pairs Ps-F/R and dsRed-F/R, followed by digested with EcoRI/BamHI and BamHI/HindlII, respectively. For site-directed mutagenesis, the primer pair Pm-F/R exchanging TCTAACAT into CGAAACAA within the extended -10 
region (Fig. 1-b) was synthesized, followed by annealing $10 \mu \mathrm{M} P \mathrm{Pm}-\mathrm{F}$ and $\mathrm{Pm}-\mathrm{R}$ at a temperature gradient from $95^{\circ} \mathrm{C}$ to $20^{\circ} \mathrm{C}$ gradually using a Biometra Thermocycler to obtain dsDNA with 5'-overhanging EcoRI and 3'- overhanging BamHI. After validation by PCR and sequencing, both reporter fusions PprnS::dsRed and PprnM::dsRed were generated through triple ligation into pUCP26 which was first digested with EcoRI/Hindll, and then transformed into E.coli S17-1. Next, both plasmids pUCP26/prnS:::dsRed and pUCP26/prnM::dsRed were separately conjugated into the wild-type G3 and $\Delta r p o S$ mutant. The promoter activities were monitored as red fluorescence signals at $535 \mathrm{~nm}$ excitation/ $595 \mathrm{~nm}$ emission, and the cell density OD 600 was measured using micro-plate reader.

Statistical analysis All data were subjected to one-way-ANOVA analysis using SPSS Statistics 21 to compare treatment mean values. Each treatment was tested in at least triplicate. Experiments were performed at least two times independently.

\section{Results}

Cloning and phylogenetic analysis of the prnABCD operon from S. plymuthica G3 The complete prnABCD operon with its promoter region was PCR amplification from G3 chromosome DNA and cloned into pMD19-T as described in the Material and Methods. The resulting recombinant plasmid pPRN was sequenced, which carries ca. 6130 bp insert including a 475 bp upstream sequence from the prnA start codon (the GenBank accession no. JF274257).The BLAST program was used to identify homologues of prnABCD in the GenBank databases.

Phylogenetic analysis of the whole prnABCD sequence from S. plymuthica $\mathrm{G} 3$ and 20 representatives of PRN-producing strains retrieved from GenBank was performed. The results (Figure S1) showed that the prnABCD operons from strain G3 and other Serratia strains are closely related to Pseudomonas spp., clustering into one clade belonging to Gammaproteobacteria, but not with Burkholderia which is a member of Betaproteobacteria. This finding suggests that the clustering pattern of prnABCD among Serratia, Pseudomonas 
and Burkholderia species reflects their taxonomic relationships.

Expression of G3 prnABCD operon and PRN identification To determine whether the G3 prnABCD operon is responsible for PRN production, two strategies were used: (a) heterologous expression of G3 prnABCD operon in E. coli DH5a, and (b) construction of a G3 conditional mutant of prnABCD under the control of the inducible Ptac promoter. For heterologous expression, the plasmid pPRN harbouring G3 prnABCD with its native promoter was introduced into E. coli DH5a competent cells by heat shock. TLC analysis showed that positive clones of the recombinant E.coli DH5a (Fig. 2, Lane 2) expressed prnABCD successfully and synthesized the end product, PRN, as indicated by the presence of a purple spot after staining with $2 \%$ Ehrlich's reagent. Synthetic PRN was used as a positive control (Fig. 2, Lane S). As expected, no PRN production was observed for the negative control E. coli DH5a/pMD19-T with the empty plasmid (data not shown). Furthermore, to genetically manipulate the PRN production levels in strain G3, an IPTG-inducible prnABCD mutant, prnind was constructed and selected, where the native promoter of the G3 prnABCD was replaced with the lac/Q Ptac inducible promoter through gene replacement and homologous recombination, which was validated by PCR and sequencing (data not shown). In the absence of the inducer IPTG, the mutant prnind should function as a prnABCD mutant. Conversely, IPTG induction should over-express prnABCD to produce higher levels of PRN than the wild-type. As expected, the TLC assay verified the above scenario by showing that the mutant prnind was defective in PRN production without IPTG induction (Fig. 2, Lane 3), but accumulated PRN when induced with $0.5 \mathrm{mmol}$ IPTG (Fig. 2, Lane 4).The wild-type G3 was used as a positive control (Fig. 2, Lane 2).

PRN is required for antifungal activity Dual culture bioassays of antifungal activity with $C$. parasitica on PDA plates showed a positive correlation between the PRN production levels and their antifungal activity among the different strains (Fig. 3). The recombinant E. coli DH5a/pPRN expressed prnABCD from G3, and secreted high levels of the end product PRN into PDA media generating an obvious inhibition zone compared with the $E$. coli 
DH5a/pMD19-T as a negative control (Fig. 3-a). Meanwhile, the prnind mutant in the presence of $0.5 \mathrm{mmol}$ IPTG (Fig. 3-b) exhibited higher antifungal activity due to PRN overproduction than the treatment in the absence of IPTG as a control (Fig. 3-c), where the expression of prnABCD was inhibited, no inhibition zone was observed. These findings suggest that PRN plays a central role in the suppression of the pathogenic fungus $C$. parasitica by strain G3.

PRN modulated swimming motility In addition to antimicrobial activity, PRN was found to modulate cell motility in strain G3. As shown in Fig. 4, in the conditional mutant prnind, 0.5 mmol IPTG induction greatly stimulated swimming ability, but the absence of IPTG decreased the swimming zone compared to the wild-type G3. Synthetic PRN at final concentration of 200 $\mathrm{ng} / \mathrm{ml}$ was used as a positive control also stimulated the swimming motility of both wild-type G3 and the inducible mutant prnind, but $0.5 \mathrm{mmol}$ IPTG had no effect on the swimming motility of the wild-type.Furthermore, no obvious difference in bacterial growth was observed between the wild type and the prnind mutant in the presence or absence of IPTG (Fig. S2), indicating that the impacts on cell motility by PRN are not due to differences in growth rate. These findings indicate that PRN, apart from being a secondary metabolite (antibiotic) with broad-spectrum antimicrobial activity, could also function as a signal molecule regulating bacterial behaviours.

Interplay between QS and RpoS regulates G3 prnABCD expression The global regulators QS and RpoS have been reported to affect PRN production in strains of $S$. plymuthica (Ovadis et al., 2004; Liu et al., 2007) or P. protegens (Sarniguet et al., 1995). To determine if this regulation generally applies, TLC or LC-MS assay was carried out to compare PRN production between the wild-type G3 and a quorum quenching strain expressing the lactonase AiiA or a $\triangle r p o S$ mutant. The results suggested that quorum quenching significantly reduced PRN production in strain G3 (Figure S3), which agrees with previous observations on the rhizospheric S. plymuthica HRO-C48 (Liu et al., 2007). Similarly, LC-MS2 analysis (Fig. 5) showed that the mutation in RpoS (peak area: $9.58 \times 10^{4}$ ) also greatly decreased the PRN yield compared to the wild-type G3 (peak area: $2.28 \times 10^{6}$ ) as observed in P. protegens Pf-5. 
This shows that both QS and RpoS positively regulated PRN biosynthesis in S. plymuthica G3. The PRN MS2 spectra are shown in Figure S4. To better understand the mechanisms behind RpoS and AHL-mediated control of PRN biosynthesis, the expression of a prnA::/ux promoter fusion in strain G3 supplemented with its major types of $\mathrm{AHL}$ signal molecules, 3-oxo-N-hexanoyl-homoserine lactone (3OC6-HSL, OHHL), the unsubstituted $\mathrm{N}$-butyryl-homoserine lactone (C4-HSL, BHL) and $\mathrm{N}$-hexanoyl-homoserine lactone (C6-HSL, $\mathrm{HHL}$ ) (Liu et al., 2010) at a final concentration of $0.5 \mu \mathrm{m}$ was performed (5 mM AHLs dissolved in methanol as stock solutions). $1 \mu \mathrm{l}$ methanol added to $10 \mathrm{ml}$ LB was used as a control, no bioluminescence difference was observed when compared with LB alone (data not shown). The results showed that $A H L$ signalling positively modulated prnABCD transcription, and the maximum activity was induced by a mixture of three types of AHLs (1: 1: 1). 30C6-HSL was the optimum single AHL signal for prnABCD transcriptional activity (Fig. 6-a). Similarly, the transcription of $r p o S:: /$ lux promoter fusion was also stimulated by $\mathrm{AHL}$ signals, and the mixture of AHLs induced the highest level of activity. In contrast to the prnA::Iux fusion, C4-HSL, not 30C6-HSL, was the optimum single signal molecule for the transcription of rpoS (Fig. 6-a). Reciprocally, RpoS also has an impact on the transcription of the luxl homologous gene spll, but not spsl (Fig. 6-b), which is known to encode the AHL synthase Spsl to produce the unsubstituted AHLs only (Liu et al., 2010).

Expression of the prnABCD operon is RpoS-dependent The expression of the prnA::/ux promoter fusion ( $500 \mathrm{bp}$ prnA upstream sequence) in the wild-type or the $\Delta r p o S$ mutant background was also measured. As shown in Fig. 7-a, the relative bioluminescence unit (RLU) of the prnA::/ux reporter fusion significantly decreased in the $\Delta r p o S$ mutant compared to the wild-type G3, suggesting that $\operatorname{prn} A B C D$ transcription might be RpoS-dependent. To validate this hypothesis, the fluorescent reporter $d s R e d$ was fused, in combination with site-directed mutagenesis of the conserved nucleotides in the extended -10 region (TCT....T replaced with CGA....A) to the shorter ( $60 \mathrm{bp})$ prnA upstream sequence. Assays of the dsRed-based reporter fusions demonstrated the ca. $60 \mathrm{bp}$ prnA upstream sequence (prnS::dsRed) does have promoter activity, but its activity decreased in the $\Delta r p o S$ mutant compared to the 
wild-type (Fig.7-b), the same trend as the prnA::/ux promoter fusion (Fig. 7-a). Meanwhile, the relative fluorescent unit (RFU) of prnM::dsRed promoter fusion in both wild-type and the $\Delta r p o S$ mutant was also greatly reduced due to mutation in the conserved nucleotide positions compared to the native prnS::dsRed (Fig. 7-b). The results indicated the prnABCD upstream sequence includes the $\sigma^{S}$-selective promoter, revealing that RpoS could directly control the transcription of $\operatorname{prn} A B C D$, in addition to affecting PRN yield. Taken together, these findings suggest that RpoS may play a key role in fine tuning both transcription and translation of the prnABCD loci through cross talk with QS, which could also have impacts on the antifungal activity and cell motility of S. plymuthica G3 (Fig. 8).

\section{Discussion}

PRN-producing strains have been described mainly in a narrow range of gram-negative bacteria of the genera Burkholderia, Pseudomonas, and Serratia, and are key players in the biocontrol of plant diseases (Hill et al., 1994; Kalbe et al., 1996; Hammer et al., 1999; Ligon et al., 2000; Costa et al., 2009). For example, PRN production from S. plymuthica was required for the suppression of a broad range of fungal pathogens, such as soil-borne Rhizoctonia solani and Pythium aphanidermatum in vegetables (Ovadis et al., 2004), airborne Botrytis cinerea and Sclerotinia sclerotiorum (Kamensky et al., 2003), and post-harvest diseases caused by Penicillium spp. in peaches and apples (de Vleeschauwer and Höfte, 2007). To improve its biocontrol efficacy, we sought to gain a better insight into PRN biosynthesis and regulation in S. plymuthica by cloning the prnABCD loci from S. plymuthica G3 into E. coli. TLC detection confirmed that the four genes $p r n A, p r n B, p r n C$ and $p r n D$ are sufficient for the production of the antibiotic PRN without the need for the surrounding genes, similar to $P$. fluorescens BL915 (Hammer et al., 1997). This finding was further verified by constructing a G3 conditional mutant prnind under the control of the inducible Ptac promoter, which behaved like a prnABCD mutant in the absence of IPTG by barely producing PRN (Fig. 2 Lane 3). More importantly, the prnind mutant induced with $0.05 \mathrm{mmol}$ IPTG also behaved like an over-expressing strain of the prnABCD operon, which has potential for PRN overproduction 
(Fig. 2, Lane 4). Correspondingly, PRN contents secreted by the wild-type G3 and its derivatives are positively correlated with their antifungal activity against $C$. parasitica (Fig. 3), consistent with previous studies in various biocontrol strains of Pseudomonas (Hill et al., 1994; Sarniguet et al., 1995), Burkhoderia (Hwang et al., 2002) and Serratia (Ovadis et al., 2004). Recently, the entire prnABCD operon from $P$. protegens Pf-5 was expressed successfully in tomato plants through the plant universal vector IL-60, resulting in the appearance of a unique plant phenotype with resistance to damping-off disease caused by $R$. solani (Mozes-Koch et al., 2012).These findings indicate that the role played by PRN in biological control of plant diseases is important and conserved in bacteria.

Apart from their antimicrobial activities, the antibiotics phenazine (Dietrich et al., 2006; Pierson et al., 2010) and 2,4-diacetylphloroglucinol (DAPG) (Maurhofer et al., 2004; Combes-Meynet et al., 2011) both serve as signal molecules to induce cross-talk among rhizoplane bacterial communities and have impacts on bacterial behaviours. Similarly, our investigation of swimming motility of the conditional mutant prnind with or without IPTG induction showed that PRN also affected cell motility which was confirmed by using a synthetic PRN standard as a positive control (Fig. 4). Thus, PRN may also function as a signal molecule, beyond its role as an antibiotic to suppress fungal pathogens. However, the non-IPTG-induced prnind could still swim; one possible explanation may be due to PRN-mediated alteration of gene expression such as motility-related genes encoding chemotaxis, flagellar etc., and/or their regulatory genes, but still to be investigated. For instance, RNA-seq based transcriptomic studies, together with qRT-PCR and phenotypic analyses could shed light on the molecular basis for PRN-driven regulation of gene expression, and unravel the reasons why PRN can affect cell motility on a whole genome scale. Altogether, the construction of the inducible mutant could be a powerful tool that can be used not only for improving the PRN yield, but also for exploring the role of PRN in bacterial physiology and behaviour. It is worth noting that the target gene or operon suitable for the construction of the Ptac inducible mutant must be a single copy with no polar effects. Fortunately, sequencing the whole genome of G3 (Liu and Chan unpublished data) allowed us to discover only one copy of the prnABCD loci in strain G3, further analysis of its genomic context by Virtual Footprint 3.0 (Münch et al., 2005) predicted a 171 bp ORF 
encoding hypothetical protein flanked the prnABCD operon, but with its own promoter approximately 50 bp upstream from the start codon ATG, and within prnD ORF. Therefore, we do not expect any polar effect on surrounding genes.

Increasing our knowledge of regulatory mechanisms governing the biosynthesis of antibiotics may help in the construction of strains with enhanced biocontrol activity (Chet and Chernin, 2002). The manipulation of bacterial regulatory systems, such as GacS/GacA TCS or alternative sigma factors in $P$. fluorescens has resulted in a significant improvement of the biocontrol potential of this organism (Schnider et al., 1995; Haas et al., 2000). QS or RpoS positively regulate PRN biosynthesis in the rhizospheric S. plymuthica HRO-C48 (Liu et al., 2007), the Burkholderia cepacia complex (Schmidt et al., 2009), and P. protegens Pf-5 (Sarniguet et al., 1995). We have provided evidence that both AHL signalling and the sigma factor RpoS can stimulate prnABCD transcription and the secretion of the end product PRN in S. plymuthica G3. Bioluminescence assay of the lux-based promoter fusions suggest that the transcription of both prnABCD and rpoS from G3 is significantly induced by the mixture of $A H L$ signals in a cell density-dependent manner under the control of QS systems (Fig. 6-a). Interestingly, mutation in RpoS also had a considerable impact on the transcription of the IuxI homologue spll, but not spsl (Fig. 6-b), which is responsible for the synthesis of unsubstituted AHLs, especially C4-HSL in strain G3 (Liu et al., 2011). These findings indicate that RpoS selectively controls the expression of SplI/SpsI QS regulators, besides the cross-link between the QS systems and RpoS, which is similar to previous reports in several Pseudomonas spp. For instance, there are two AHL-mediated QS systems, LasIR/RhlIR, in P. aeruginosa, and RpoS negatively regulates QS through repression of the transcription of rhll, but not lasl; on the other hand, QS activates rpoS expression through RhIIR (Venturi 2006). Further sequence comparison revealed a potential $\sigma^{S}$ extended -10 region approximately 30 bp upstream of the prnA start codon which was verified through site-directed mutagenesis together with dsRed-based promoter fusion assays (Fig. 7-b). To the best of our knowledge, this is the first experimental evidence showing that $\operatorname{prn} A B C D$ is an RpoS-dependent operon; therefore, $\mathrm{QS}$ may at least indirectly affect PRN biosynthesis via RpoS-driven direct control of the transcription of prnABCD since no lux box-like element was found in the upstream sequence of 


\section{Acknowledgements}

We would like to thank N. Halliday for kind assistance with LC-MS analysis.

Funding: The study was funded partially by the National Natural Science Foundation of China (grant no. 31240046), EU Marie Curie IIF project PROAGROBAC (grant no. 297882) and the Special Fund for Agro-Scientific Research in the Public Interest of China (grant no. 201503110-12).

\section{Compliance with ethical standards}

Conflict of Interest: All authors declare that he/she has no conflict of interest.

Ethical Statement: This article does not contain any studies with human participants or animals performed by any of the authors 


\section{References}

Arima K, Imanaka H, Kousaka M, Fukuda A, Tamura G (1964) Pyrrolnitrin, a new antibiotic substance, produced by Pseudomonas. Agric Biol Chem 28: 575-576.

Atkinson S, Chang CY, Patrick HL, Buckley CM, Y Wang, Sockett RE, Cámara M, Williams P (2008) Functional interplay between the Yersinia pseudotuberculosis YpsRI and YtbRI quorum sensing systems modulates swimming motility by controlling expression of flhDC and fliA. Mol Microbiol 69: 137-151.

Ausubel FM, BrentR, Kingston RE, Moore DD, Seidman JG, Smith JA, Struhl K (1994) Current protocols in molecular biology. John Wiley \& Sons Inc., NY.

Becker G, Hengge-Aronis R (2001) What makes an Escherichia coli promoter sigma(S) dependent? Role of the -13/-14 nucleotide promoter positions and region 2.5 of sigma (S). Mol Microbiol 39(5):1153-65.

Chernin L, Brandis A, Ismailov Z, Chet I (1996) Pyrrolnitrin production by an Enterobacter agglomerans strain with a broad spectrum of antagonistic activity towards fungal and bacterial phytopathogens. Curr Microbiol 32: 208-212.

Chet I, Chernin L (2002) Biocontrol, microbiol agents in soil. In: Bitton G (ed) Encyclopedia of environmental microbiology, Join Willey \& Sons Inc., New York, pp 450-465.

Combes-Meynet E, Pothier JF, Moënne-Loccoz Y, Prigent-Combaret C (2011) The Pseudomonas secondary metabolite 2,4-diacetylphloroglucinol is a signal inducing rhizoplane expression of Azospirillum genes involved in plant-growth promotion. Mol Plant Microbe Interact 24(2):271-284.

Costa R, van Aarle IM, Mendes R, van Elsas JD (2009) Genomics of pyrrolnitrin biosynthetic loci: evidence for conservation and whole-operon mobility within gram-negative bacteria. Environ Microbiol 211:159-175.

Dennis JJ, Zylstra GJ (1998) Plasposons: modular self-cloning minitransposon derivatives for rapid genetic analysis of gram-negative bacterial genomes. Appl Environ Microbiol 64: $2710-2715$.

de Vleeschauwer D, Höfte M (2007) Using Serratia plymuthica to control fungal pathogens of 
plant. CAB Rev 2: 046.

Dennis JJ and Zylstra GJ (1998) Plasposons: modular self-cloning minitransposon derivatives for rapid genetic analysis of gram-negative bacterial genomes. Appl Environ Microbiol 64:2710-2715.

Di Santo R, Costi R, Artico M, Massa S, Lampis G, Deidda D, Pompei R (1998) Pyrrolnitrin and related pyrroles endowed with antibacterial activities against Mycobacterium tuberculosis. Bioorg Med Chem Lett 8(20):2931-2936.

Dietrich LE, Price-Whelan A, Petersen A, Whiteley M, Newman DK (2006) The phenazine pyocyanin is a terminal signalling factor in the quorum sensing network of Pseudomonas aeruginosa. Mol Microbiol 61(5):1308-21.

Hammer PE, Hill DS, Lam ST, Van Pée KH, Ligon JM (1997) Four genes from Pseudomonas fluorescens that encode the biosynthesis of pyrrolnitrin. Appl Environ Microbiol 63: 2147-2154. Hammer PE, Burd W, HillDS, LigonJM, van Pée K-H (1999) Conservation of the pyrrolnitrin biosynthetic gene cluster among six pyrrolnitrin-producing strains. FEMS Microbiol Lett 180: $39-44$.

Haas D, Blumer C, Keel C (2000) Biocontrol ability of fluorescent pseudomonads genetically dissected: importance of positive feedback regulation. Curr Opinion Biotechnol 11:290-297. Heeb S, Blume C, Haas D. (2002) Regulatory RNA as mediator in GacA/RsmA-dependent global control of exoproduct formation in Pseudomonas fluorescens CHA0. J Bacteriol 184:1046-1056.

Hill DS, Stein JI, Torkewitz NR, Morse AM, Howell CR, Pachlatko JP, BeckerJO, Ligon JM (1994) Cloning of genes involved in the synthesis of pyrrolnitrin from Pseudomonas fluorescens and role of pyrrolnitrin synthesis in biological control of plant disease. Appl Environ Microbiol 60: 78-85.

Hwang J, Chilton WS, Benson DM (2002) Pyrrolnitrin production by Burkholderia cepacia and biocontrol of Rhizoctonia stem rot of poinsettia. Biol Control 25: 56-63.

Kalbe C, Marten P, Berg G (1996) Strains of the genus Serratia as beneficial rhizobacteria of oilseed rape. Microbiol Res 151: 4400-4433.

Kamensky M, Ovadis M, Chet I, Chernin L (2003) Soil-borne strain IC14 of Serratia plymuthica 
with multiple mechanisms of antifungal activity provides biocontrol of Botrytis cinerea and Sclerotinia sclerotiomm diseases. Soil Biol Biochem 35: 323-331.

Keum YS, Lee YJ, Lee YH, Kim JH (2009) Effects of nutrients on quorum signals and secondary metabolite productions of Burkholderia sp. O33. J Microbiol Biotechnol 19: $1142-1149$.

Kim CH, Kim YH, Anderson AJ, Kim YC (2014) Proteomic Analysis of a global regulator GacS sensor kinase in the Rhizobacterium, Pseudomonas chlororaphis O6. Plant Pathol $\mathrm{J}$ 30(2):220-7.

Kirner S, Hammer PE, Hill DS, Altmann A, Fischer I, Weislo LJ, Lanahan M, van PéeK-H, Ligon JM, Hill DS, Hammer PE, Torkewitz NR, Hofmann D, Kempf H, van Pee K (2000) Natural products with antifungal activity from Pseudomonas biocontrol bacteria. Pest Manage Sci 56: 688-695.

Liu X, Bimerew M, Ma YX, Müller H, Ovadis M, Eberl L, Berg G, Chernin L (2007) Quorum-sensing signaling is required for production of the antibiotic pyrrolnitrin in a rhizospheric biocontrol strain of Serratia plymuthica. FEMS Microbiol Lett 270: 299-305.

Liu X, Jia J, Atkinson S, CámaraM, Gao K, Li H, Cao J (2010) Biocontrol potential of an endophytic Serratia sp. G3 and its mode of action. World J Microbiol Biotechnol 26: 465-471. Liu X, Jia J, Popat R, Ortori CA, Li J, Diggle SP, Gao K, Cámara M (2011) Characterisation of two quorum sensing systems in the endophytic Serratia plymuthica strain G3: differential control of motility and biofilm formation according to life-style. BMC Microbiol 11: 26.

Liu X, Wu Y, Chen Y, Xu F, Halliday N, Gao K, Chan KG, Cámara M (2016) RpoS differentially affects the general stress response and biofilm formation in the endophytic Serratia plymuthica G3. Res Microbiol 167(3):168-177.

Maurhofer M, Baehler E, Notz R, Martinez V, Keel C (2004) Cross talk between 2,4-diacetylphloroglucinol-producing biocontrol pseudomonads on wheat roots. Appl Environ Microbiol 70(4):1990-1998.

Milton, D.L., O'Toole, R., Horstedt, P., and Wolf-Watz, H (1996) Flagellin A is essential for the virulence of Vibrio anguillarum. J Bacteriol 178: 1310-1319.

Mozes-Koch R, Gover O, Tanne E, Peretz Y, Maori E, Chernin L, Sela I (2012) Expression of 
an entire bacterial operon in plants. Plant Physiol 158(4):1883-1892.

Münch R, Hiller K, Grote A, Scheer M, Klein J, Schobert M, Jahn D (2005) Virtual Footprint and PRODORIC: an integrative framework for regulon prediction in prokaryotes. Bioinformatics 21: 4187-4189

Nandi M, Selin C, Brassinga AK, Belmonte MF, Fernando WG, Loewen PC, de Kievit TR (2015) Pyrrolnitrin and hydrogen cyanide production by Pseudomonas chlororaphis strain PA23 exhibits nematicidal and repellent activity against Caenorhabditis elegans. PLoS One. 10(4):e0123184. doi: 10.1371/journal.pone.0123184.

Ovadis M, Liu X, Gavriel S, Ismailov Z, Chet I, Chernin L (2004) The global regulator genes from biocontrol strain Serratia plymuthica IC1270: cloning, sequencing, and functional studies. J Bacteriol 186: 4986-4993.

Park JY, Oh SA, Anderson AJ, NeiswenderJ, Kim JC, Kim YC (2011) Production of the antifungal compounds phenazine and pyrrolnitrin from Pseudomonas chlororaphis $\mathrm{O} 6$ is differentially regulated by glucose. Lett Appl Microbiol 52: 532-537.

Pierson LS 3rd, Pierson EA (2010) Metabolism and function of phenazines in bacteria: impacts on the behavior of bacteria in the environment and biotechnological processes. Appl Microbiol Biotechnol 86(6):1659-1670.

Rampioni G, Pustelny C, Fletcher MP, Wright VJ, Bruce M, Rumbaugh KP, Heeb S, Cámara M, Williams P (2010) Transcriptomic analysis reveals a global alkyl-quinolone-independent regulatory role for PqsE in facilitating the environmental adaptation of Pseudomonas aeruginosa to plant and animal hosts. Environ Microbiol 12(6):1659-1673.

Rutherford ST and Bassler BL (2012) Bacterial quorum sensing: Its role in virulence and possibilities for its control. Cold Spring Harb Perspect Med 2(11): a012427.

Sarniguet A, Kraus J, Henkels MD, Muehlchen AM, Loper JE (1995) The sigma factor sigma s affects antibiotic production and biological control activity of Pseudomonas fluorescens Pf-5. Proc Natl Acad Sci U S A 92(26):12255-9.

Schmidt S, Blom JF, Pernthaler J, Berg G, Baldwin A, Mahenthiralingam E, Eberl L (2009) Production of the antifungal compound pyrrolnitrin is quorum sensing-regulated in members of the Burkholderia cepacia complex. Environ Microbiol 11:1422-1437. 
Schnider U, Keel C, Blumer C, Troxler J, Défago G, Haas D (1995) Amplification of the housekeeping sigma factor in Pseudomonas fluorescens CHAO enhances antibiotic production and improves biocontrol abilities. J Bacteriol 177(18): 5387-5392.

Umarov RK, Solovyev VV (2017) Prediction of prokaryotic and eukaryotic promoters using convolutional deep learning neural networks. PLoS ONE, 0171410.

Venturi V (2006) Regulation of quorum sensing in Pseudomonas. FEMS Microbiol Rev $30: 274-291$

Weber H, Polen T, Heuveling J, Wendisch VF, Hengge R (2005) Genome-wide analysis of the general stress response network in Escherichia coli: sigma S-dependent genes, promoters, and sigma factor selectivity. J Bacteriol 187(5):1591-603.

West SE, Schweizer HP, Dall C, Sample AK, Runyen-Janecky LJ (1994) Construction of improved Escherichia-Pseudomonas shuttle vectors derived from pUC18/19 and sequence of the region required for their replication in Pseudomonas aeruginosa. Gene 148: 81-86.

Zhou M, Gao K, Zeng J, Yu X, Wu Y, Ge J, Duan Y, Liu X (2012) Role of the RNA-binding protein Hfq in Serratia plymuthica. Front Biosci E4:1263-1275.

\section{Tables and Figures' Legends}

Table 1 Bacterial strains and plasmids used in this study

Table 2 Primers used in this study

Fig. 1 Schematic representation of the prnABCD loci in S. plymuthica G3 wild-type and its IPTG-inducible mutant prnind (A); and the prnA upstream DNA sequence (B)

Panel a: The gentamycin $(\mathrm{Gm})$ cassette is excised with BamHI from plasmid p34S-Gm, and the lac ${ }^{\mathcal{Q}}$ repressor with the Ptac promoter is derived from plasmid pME6032. The organization of the prnABCD operon is shown, which is followed by a 171 bp hypothetical protein (hp) with its own promoter $\mathrm{P}_{h p}$ located in the prnD ORF. Panel b: The putative $\sigma^{70}-10$ box is indicated by a black bar, and the putative $\sigma^{S}$ extended -10 region in grey box, and the conserved nucleotides in bold are replaced (short vertical lines) through site-directed mutagenesis, the primer pairs Ps-F/R are underlined. The proposed ribosome binding site (RBS) and the ATG start codon are in grey box. 


\section{Fig. 2 TLC detection of PRN production}

Lane S: synthetic PRN standard (4 $\mu$ from Sigma); Lane 1: wild-type G3; Lane 2: E. coli DH5a/pPRN carrying pMD19-T-prnABCD; Lane 3: non-IPTG induced mutant prnind ; Lane 4: prnind + 0.5 mmol IPTG

Fig. 3 Effects of the PRN production on antifungal activity in dual cultures with the pathogenic C. parasitica.

Panel a: the recombinant E. coli DH5a/pPRN carrying the G3 prnABCD operon (A); E.coli DH5a/pMD19-T with the empty plasmid as a negative control (B); and the wild-type G3 as a positive control. Panel b: IPTG induced mutant prnind. Panel C: non-IPTG induced mutant prnind.

\section{Fig. 4 Effects of the PRN production on swimming motility}

$0.5 \mathrm{mmol}$ IPTG was used for induction of the Ptac promoter expression ; $2 \mu \mathrm{l}$ of overnight bacterial suspension was inoculated on swimming plates containing $0.2 \mu \mathrm{g} / \mathrm{ml}$ concentration of synthetic PRN and incubated at $30^{\circ} \mathrm{C}$ for $16 \mathrm{~h} .2 \mu \mathrm{l}$ methanol $(\mathrm{MeOH})$ as the solvent control.

\section{Fig. 5 LC-MS2 identification of PRN production}

Panel a: HPLC profile of $10 \mu \mathrm{m}$ synthetic PRN as a positive control. Panel b: HPLC profiles of PRN extracts from the wild-type $\mathrm{G} 3$ in black and the mutant $\Delta r p o S$ in grey, respectively.

\section{Fig.6 Assay of lux-based promoter fusions}

Panel a: Impacts of $0.5 \mu \mathrm{M}$ AHLs on the transcription of prnABCD and rpoS in strain G3. Panel b: Impacts of RpoS on the transcription of the luxl homologues spll and spsl in strain G3. 5 mM AHLs dissolved in $\mathrm{MeOH}$ were used as stock solutions, $1 \mu \mathrm{l} \mathrm{MeOH}$ diluted with $10 \mathrm{ml} \mathrm{LB}$ as a control. BHL: N-butyryl-homoserine lactone (C4-HSL); HHL: $N$-hexanoyl-homoserine lactone (C6-HSL); OHHL: 3-oxo-N-hexanoyl-homoserine lactone (3OC6-HSL); Mix: the mixture of BHL, HHL and OHHL (1:1:1).

Fig. 7 RpoS-dependent transcription of the prnABCD operon

Panel a: Assay of lux-based promoter fusion to prnA. Panel b: RpoS directly activates the prnA 
transcription determined by site-directed mutagenesis.

Fig. 8 Schematic model describing the interplay between AHL signaling and RpoS involved in regulation of PRN biosynthesis in S. plymuthica G3

Supplementary materials (Figure S1-S4) 
Table 1 Bacterial strains, plasmids and primers used in this study

\begin{tabular}{|c|c|c|}
\hline Strain or plasmid & Description & Reference \\
\hline S. plymuthica G3 & Wild type, Rif ${ }^{R}$ & Liu et al. 2011 \\
\hline prnind & $\begin{array}{l}\text { G3 derivative in which prnABCD expression is under the control of } \\
\text { lac/a Ptac inducible promoter, } \mathrm{Gm}^{\mathrm{R}}\end{array}$ & This study \\
\hline$\Delta r p o s$ & A rpoS: :Km mutant of $\mathrm{G} 3, \mathrm{Km}^{\mathrm{R}}$ & Liu et al., 2016 \\
\hline E. coil DH5a & A host strain for cloning & Ausubel et al., 1994 \\
\hline E. coil S17-1 & Pro thi hsdR recA-Tpr Smr; chromosome::RP4-2 Tc::Mu-Km::Tn7 & Ausubel et al., 1994 \\
\hline pMD19-T & Cloning vector, $\mathrm{Amp}^{\mathrm{R}}$ & Takara \\
\hline pPRN & Plasmid pMD19-T harboring G3 prnABCD operon & This study \\
\hline pME6032 & Shuttle vector with lac ${ }^{\mathrm{P}} \mathrm{P}$ tac inducible promoter; $\mathrm{Tc}^{\mathrm{R}}$ & Heeb et al., 2002 \\
\hline p34S-Gm & Source of $\mathrm{Gm}^{\mathrm{R}}$ cassette & Dennis et al., 1998 \\
\hline pBluescript II SK(-) & Cloning vector, $\mathrm{Amp}^{\mathrm{R}}$ & Stratagene \\
\hline pDM4 & Suicide vector; sacBR, oriR6K; $\mathrm{Cm}^{\mathrm{R}}$ & Milton et al. 1996 \\
\hline pDM4-YX & pDM4 carrying lacl ${ }^{Q}$ Ptac-prnA, $\mathrm{Gm}^{\mathrm{R}}, \mathrm{Cm}^{\mathrm{R}}$ & This study \\
\hline pUCP26 & Broad-host-range cloning vector; $\mathrm{Tc}^{\mathrm{R}}$ & West et al., 1994 \\
\hline pBluelux & a promoter-less luxCDABE cassette in pBluescript II, $A m p^{R}$ & Atkinson et al., 2008 \\
\hline pPprnA::/ux & pUCP26 with a prnABCD promoter fusion to $l u x C D A B E, \mathrm{Tc}^{\mathrm{R}}$ & Zhou et al., 2012 \\
\hline pPrpoS::/ux & pUCP26 with a rpoS promoter fusion to luxCDABE, $\mathrm{Tc}^{\mathrm{R}}$ & This study \\
\hline pPspll::/ux & pUCP26 with a sp/l promoter fusion to luxCDABE, $\mathrm{Tc}^{\mathrm{R}}$ & This study \\
\hline pPspsl::lux & pUCP26 with a sps/ promoter fusion to luxCDABE, $\mathrm{Tc}^{\mathrm{R}}$ & This study \\
\hline pDsRed2 & pDsRed-express2 vector, Amp ${ }^{R}$ & Clontech \\
\hline pPprnS::dsRed & pUCP26 carrying a shorter prnA promoter with a -10 box, $\mathrm{Tc}^{\mathrm{R}}$ & This study \\
\hline pPprnM::dsRed & pUCP26 carrying mutation in -10 box of the prnA promoter, $\mathrm{Tc}^{\mathrm{R}}$ & This study \\
\hline
\end{tabular}


Table 2 Primers used in this study

\begin{tabular}{|c|c|}
\hline Primer & 5'-3' sequences \\
\hline prnA-F & GGTGTTCGATTTATAGGGT \\
\hline prnD-R & CGCCATGATGACAGTGA \\
\hline prnind $_{1}-\mathrm{F}$ & CGAGCTCTTTCCGAATGTTGTTGA (Sacl) \\
\hline prnind $_{1}-\mathrm{R}$ & CGGGATCCTTGCTCATGACACTCT (BamHI) \\
\hline prnind $_{2}-\mathrm{F}$ & GGGAATTCATGAGCAAACCGATC (EcoRI) \\
\hline prnind $_{2}-\mathrm{R}$ & TTGATGGAGTCAGAGAG \\
\hline prn-luxF & CGTAAGTAACGAATGAATC \\
\hline prn-luxR & CAGGCTAGACTCTCGTCT \\
\hline rpos-luxF & GAATTCACTGCAACGGTTGATTCT (ECORI) \\
\hline rpos-luxR & ATACCGCGAGCAGAATATC \\
\hline spll-luxF & CAGCGACTTCGACAGCAT \\
\hline spll-luxR & TTGGCGCAAATATATAGCG \\
\hline spsl-luxF & TGTATTGGTCGGTGGTGA \\
\hline spsl-luxR & GTCTTTCGGTATTGGTGAGT \\
\hline Ps-F & GAATTCAGAGTCTAGCCTGATTAGAAC (EcoRI) \\
\hline Ps-R & GGATCC ATAGGAGGATAGTGGAGATG (BamHI) \\
\hline Pm-F & AATTCAGAGTCTAGCCTGATTAGAACCTAACGAAACAACTCCACTATCCTCCTATG $($ ECoRI) \\
\hline Pm-R & GATCCATAGGAGGATAGTGGAGTTGTTTCGTTAGGTTCTAATCAGGCTAGACTCT $\underline{\text { (BamHI) }}$ \\
\hline dsRed-F & GGATCCAGGAAACAGATGGATAGCACTGAGAACGT (BamHI, RBS) \\
\hline dsRed-R & AAGCTTCTACTGGAACAGGTGGTG(HindIII) \\
\hline
\end{tabular}

* Restriction sites are underlined, Ribosome binding site RBS in bold. Annealing the primer pair Pm-F/Pm-R was completed from 95 to $20^{\circ} \mathrm{C}$ gradually in Biometra gradient PCR apparatus to obtain dsDNA with $5^{\prime}$-EcoRl and 3-BamHI cohesive end (in shade), respectively for triple ligation. 

a

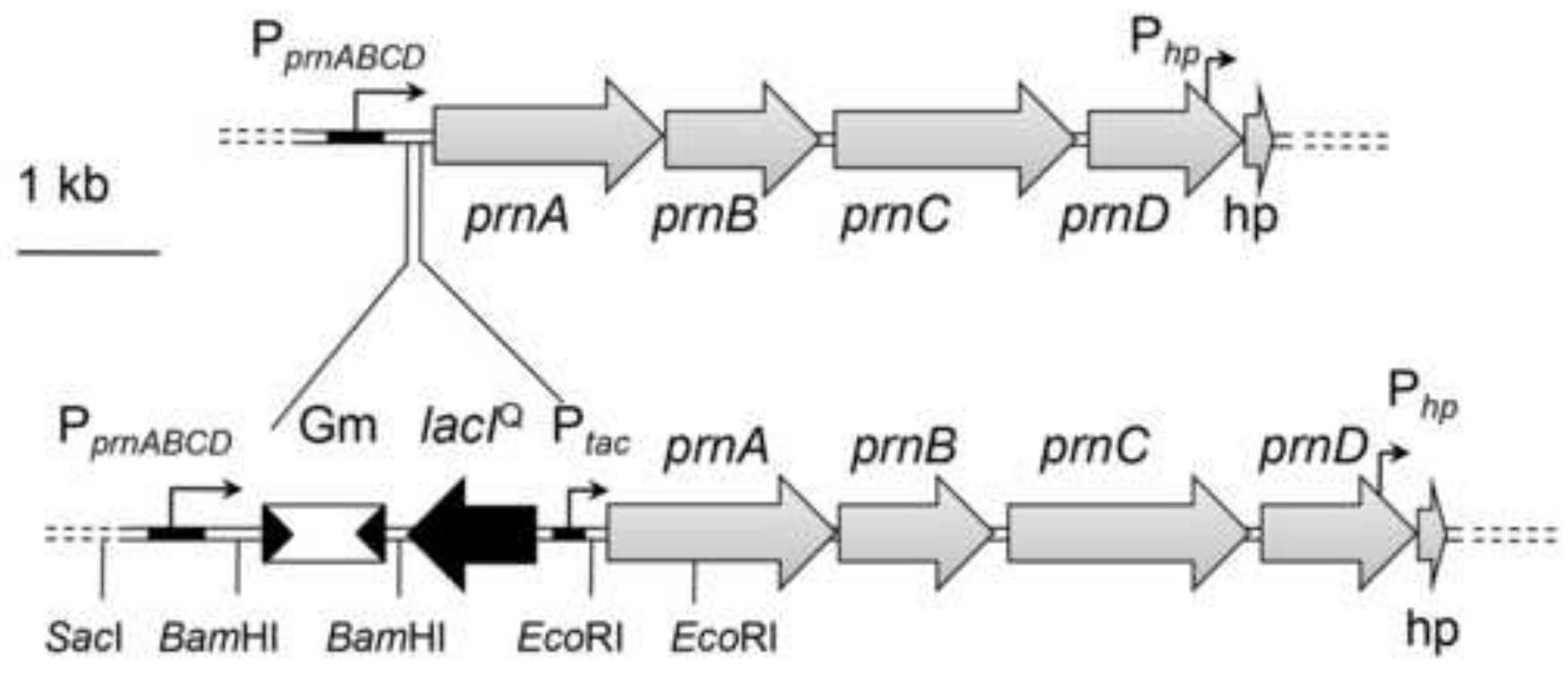

b

GATATATCGCTGAGTTAATTCTAAAACGGAATGTGGTAATTTTCAGACGAGAGTCTAGCCTGATTAGAACC

$\sigma^{s}$ extended -10 box

RBS prnA start

$\rightarrow$ Primer Ps-F

CGA A

TAATCTAACATCTCCACTATCCTCCTATGGGAGAGTGTCATGAGCAAA

$\sigma^{70}-10$ box

Primer Ps-R

$M$ S $K$ 


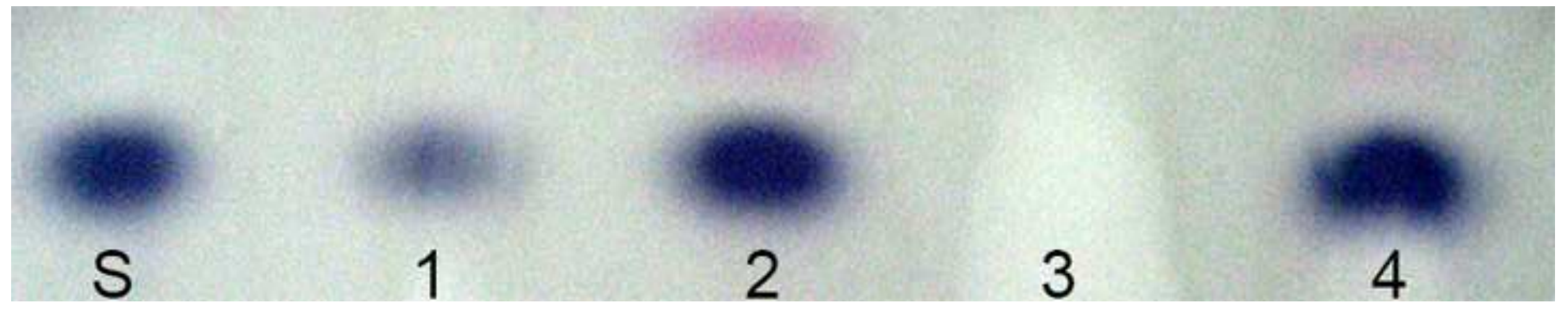




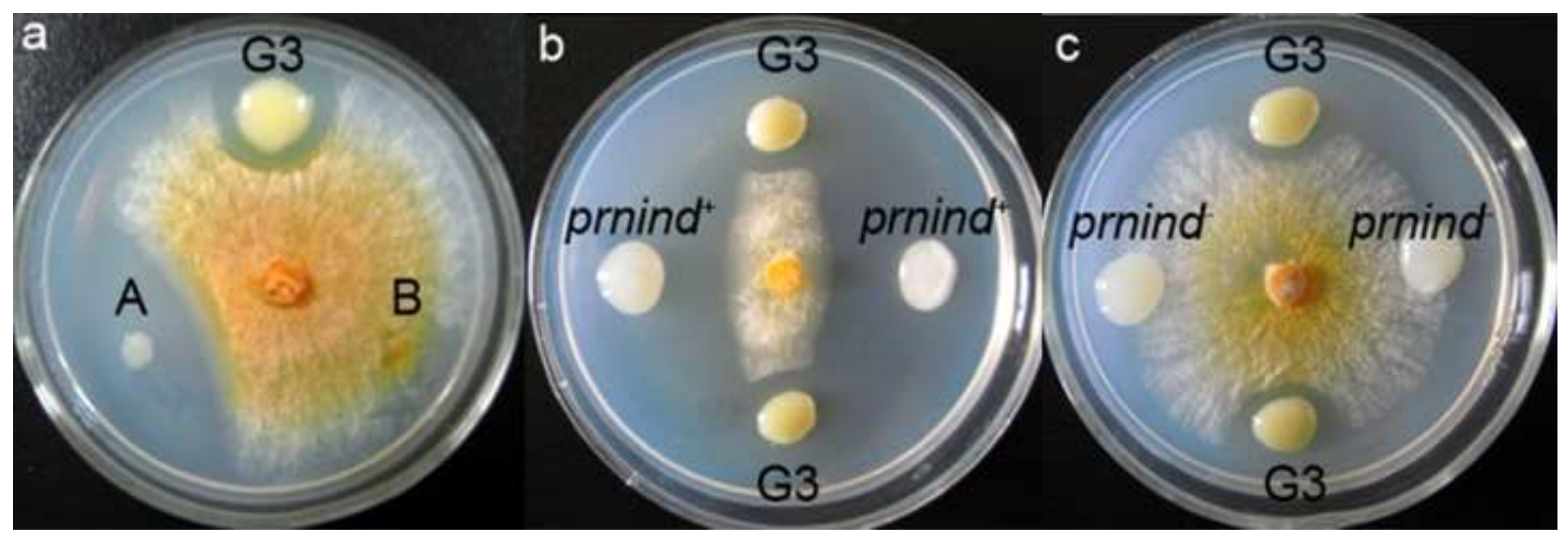




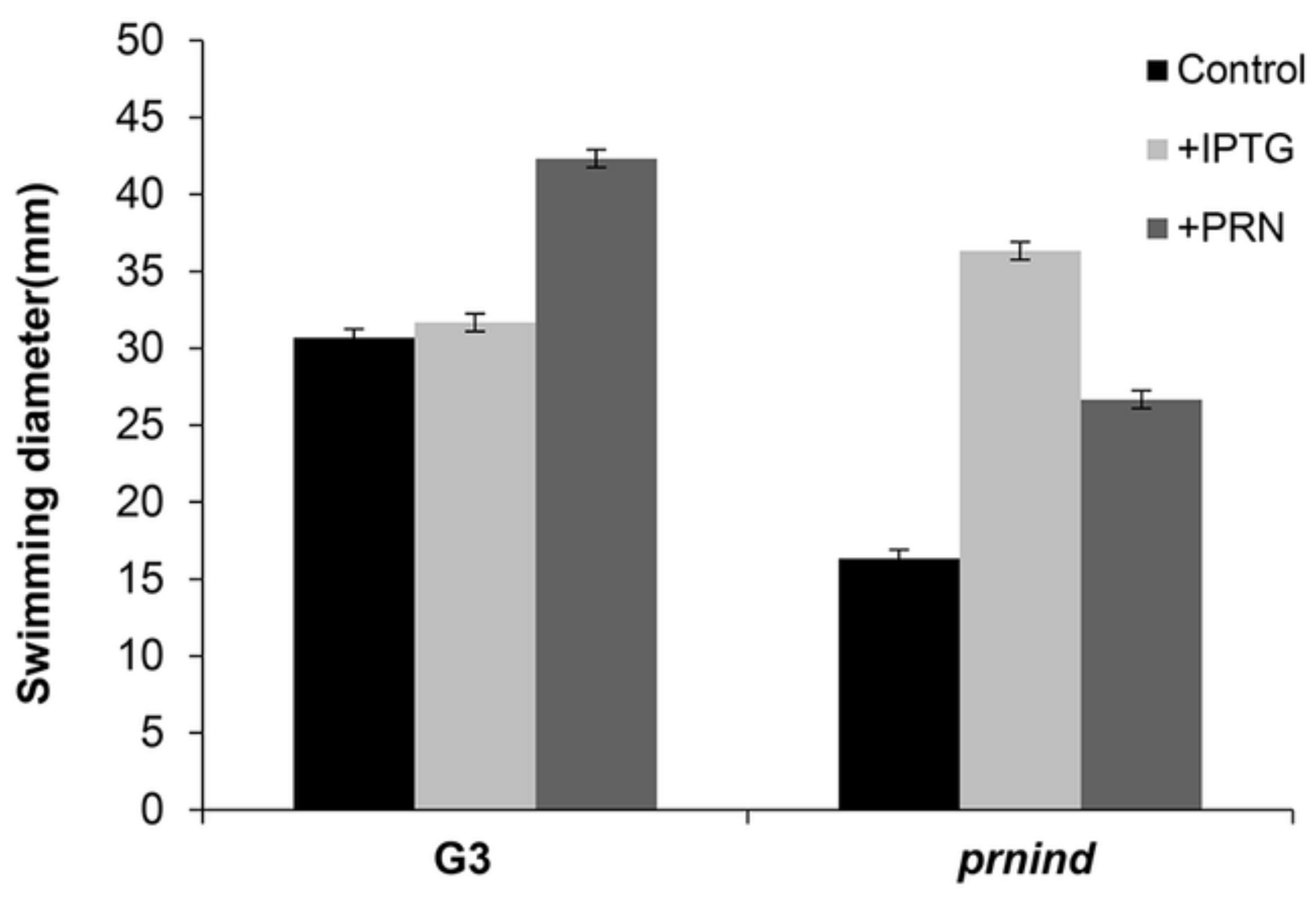




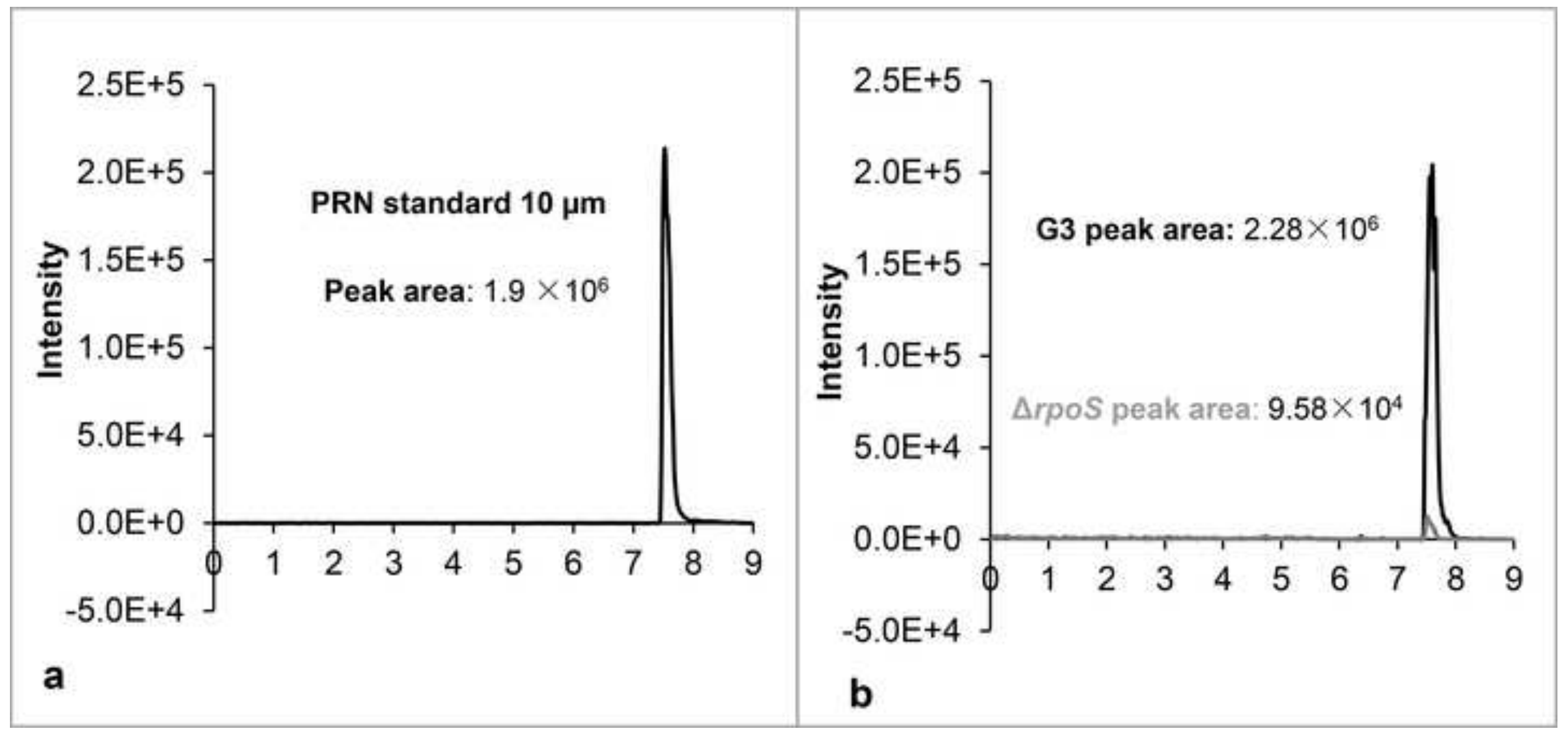




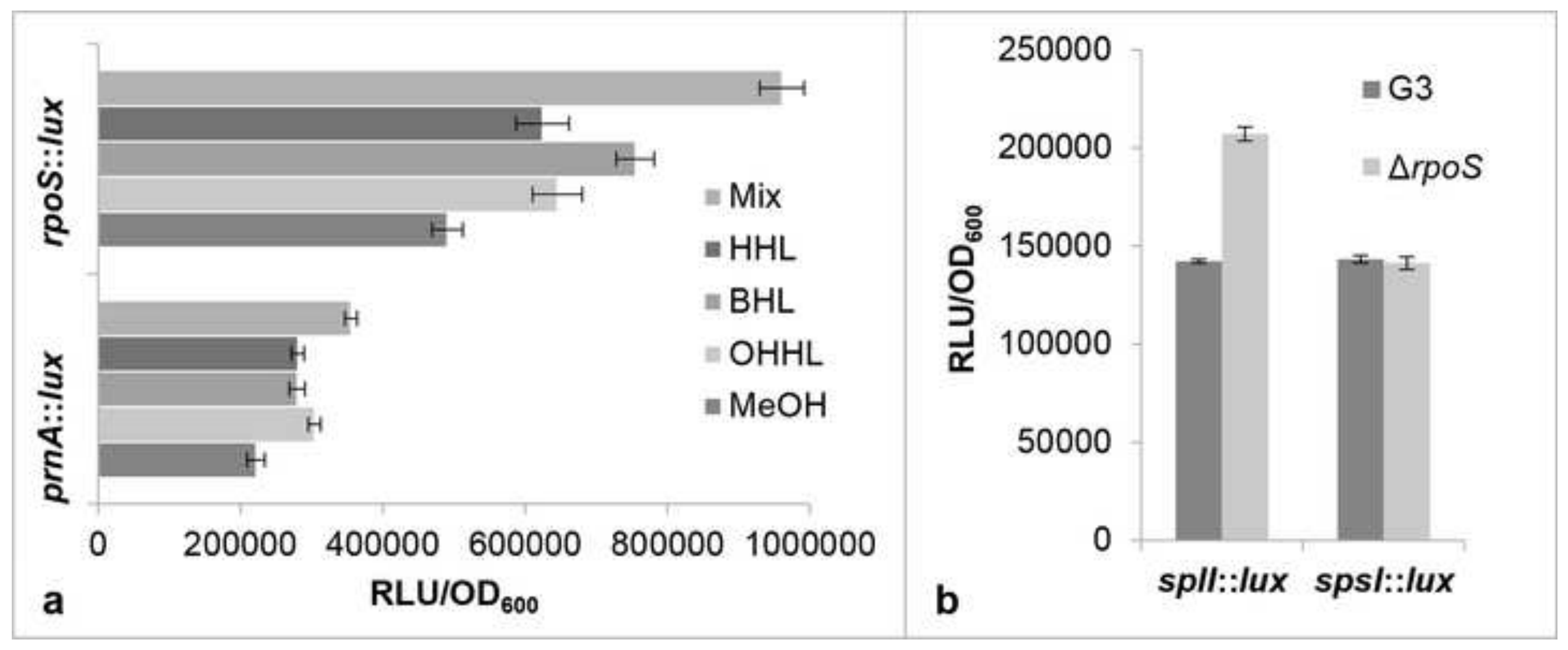




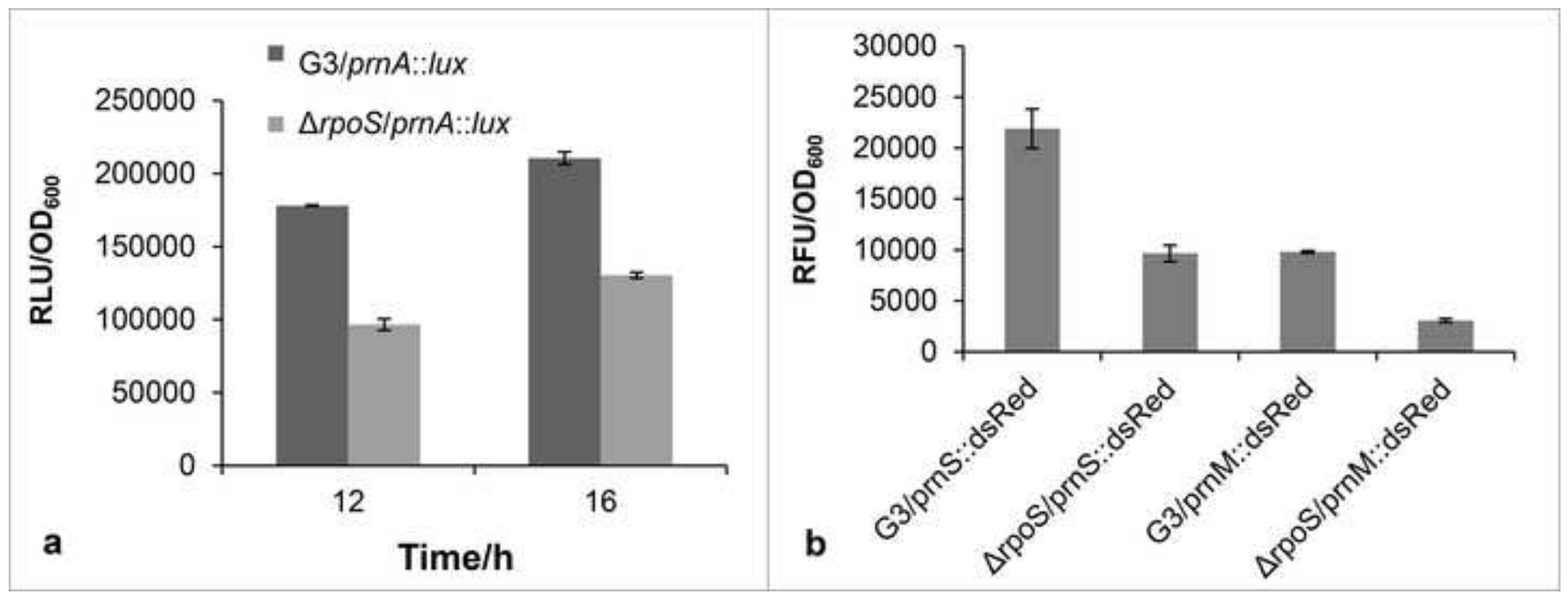




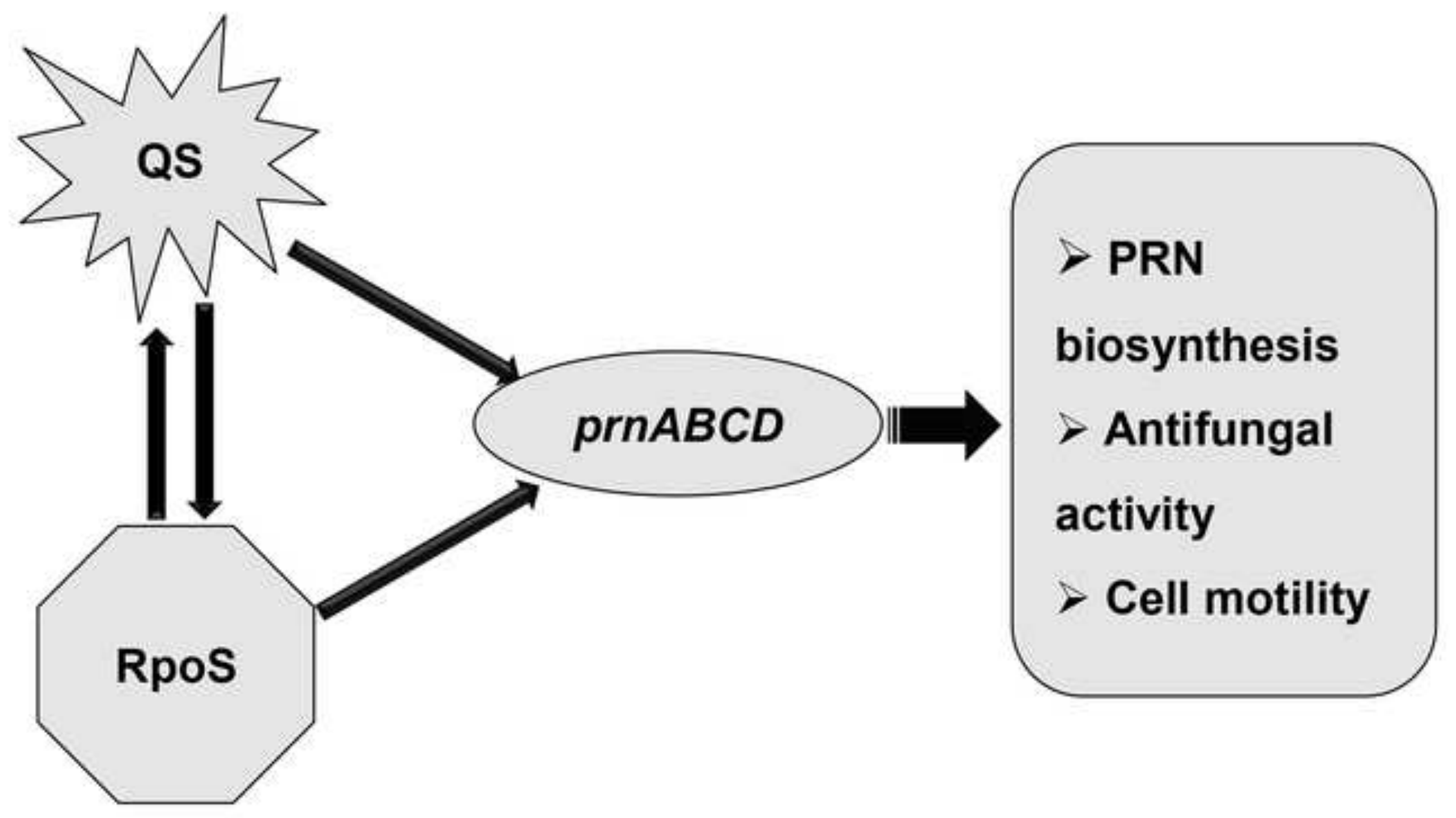


Click here to access/download Supplementary Material Supplementary materials.pdf 